\title{
Study of MV-algebras via derivations
}

\author{
Jun Tao Wang, Yan Hong She and Ting Qian
}

\begin{abstract}
The main goal of this paper is to give some representations of MValgebras in terms of derivations. In this paper, we investigate some properties of implicative and difference derivations and give their characterizations in MV-algebras. Then, we show that every Boolean algebra (idempotent MV-algebra) is isomorphic to the algebra of all implicative derivations and obtain that a direct product representation of $\mathrm{MV}$-algebra by implicative derivations. Moreover, we prove that regular implicative and difference derivations on MV-algebras are in one to one correspondence and show that the relationship between the regular derivation pair $(d, g)$ and the Galois connection, where $d$ and $g$ are regular difference and implicative derivation on $L$, respectively. Finally, we obtain that regular difference derivations coincide with direct product decompositions of MV-algebras.
\end{abstract}

\section{Introduction}

Fuzzy logic takes the advantage of the classical logic to handle uncertain information and fuzzy information. In recent decades, various logical algebras have been proposed as the semantical systems of fuzzy logic systems, for example, MV-algebras [3], BL-algebras [10] and MTL-algebras [7]. Among these logical algebras, MV-algebras are very important algebraic structures. MValgebras were introduced in [3] as an algebraic counterpart of the Eukasiewicz infinite valued propositional logic, but their theories were also developed from

Key Words: MV-algebra, implicative derivation, difference derivation, Boolean algebra. 2010 Mathematics Subject Classification: Primary 06D35; Secondary 03G05.

Received: 05.12.2018

Revised: 20.03 .2019

Accepted: 31.03 .2019 
an algebraic point of view $[3,4,5,6,17]$. It is generally known that MValgebras can also be studied within the context of abelian lattice-ordered groups with strong units ( $\ell$-groups), this viewpoint was made possible by the fundamental results of Mundici $[5,15]$ that the category of MV-algebras is equivalent to the category of $\ell$-groups.

The notion of derivations, introduced from the analytic theory, is helpful for studying algebraic structures and properties in algebraic systems. In 1957, Posner [16] introduced the notion of derivations in a prime ring $(R,+, \cdot)$, which is a mapping $d: R \longrightarrow R$ satisfying the following conditions:

$$
\text { (i) } d(x+y)=d(x)+d(y) \text {, (ii) } d(x \cdot y)=(d(x) \cdot y)+(x \cdot d(y)) \text {, }
$$

for any $x, y \in R$, and gave some characterizations of commutative prime ring in terms of derivations. Subsequently, Jun [12] applied the notion of derivations to BCI-algebras and gave characterizations of p-semisimple BCI-algebra by regular derivations. Inspired by this, several authors have studied generalized derivations in BCI-algebras [2, 23]. In the past few years, Xin [19, 20] introduced the concept of derivations in a lattice, where operations + and . are interpreted as lattice operations $\vee$ and $\wedge$, respectively, and characterized modular lattices and distributive lattices by isotone derivations; Alshehri [1] introduced the notion of (additive) derivations for an MV-algebra, where operations + and $\cdot$ are interpreted as $\oplus$ and $\odot$, and discussed some related properties; Sang and Yong $[13,21]$ investigate derivation and generalized derivation in lattice implication algebra and characterized the fixed set by these derivations; He [11] investigated derivations in residuated lattices and characterize Heyting algebras in terms of derivations; Zhu [22] introduced some derivations in linguistic truth-valued lattice implication algebras and discussed the relationship between them; Wang [18] investigated derivations in commutative multiplicative semilattices and characterize quantales in terms of derivations; Liang [14] introduced the notion of derivations of EQ-algebra and gave some characterizations of them.

In this paper, we will further study derivations of MV-algebras. One of our aims is to obtain some representations of MV-algebras by means of derivations. In particular, we will obtain the following main results: (1) every Boolean algebra is isomorphic to the algebra of all implicative derivations, which shows that the structure of a Boolean algebra is completely determined by its set of all implicative derivations. Indeed, this results essentially go a step further of the following important results in [19]: every distributive lattice is isomorphic to the algebra of all principal lattice derivations; (2) there exists a one to one correspondence between regular difference derivations and direct product decompositions on MV-algebras, which shows that regular difference derivations coincide with direct product decompositions of MV-algebras. It's always been 
known that Galois connections play a central role in studying MV-algebras, and so the relationship among implicative, difference derivations and Galois connections is the other aim of us to study. Indeed, we will get the following main results: (3) there exists an antitone Galois connection between the set of implicative and difference derivations; (4) regular implicative and difference derivations on MV-algebras are in one to one correspondence; (5) every regular derivation pair $(d, g)$ is a Galois connection on $L$ if and only if $g(L)=d(L)$.

The paper is organized as follows: In Section 2, we review some basic definitions and results about MV-algebras. In Section 3, we give some representations of MV-algebra in terms of implicative derivations. In Section 4, we discuss the relationship between derivations and Galois connections.

\section{Preliminaries}

In this section, we summarize some results about MV-algebras, which will be used in the following sections.

An $M V$-algebra is an algebraic structure $L=(L, \oplus, *, 0)$ satisifying the following axioms: for any $x, y \in L$,

(MV1) $(L, \oplus, 0)$ is a commutative monoid,

(MV2) $\left(x^{*}\right)^{*}=x$,

(MV3) $0^{*} \oplus x=0^{*}$,

(MV4) $\left(x^{*} \oplus y\right)^{*} \oplus y=\left(y^{*} \oplus x\right)^{*} \oplus x$.

We shall adopt the usual conventions for MV-terms:* operation is more binding than $\oplus$. On MV-algebra $L$, we define the constant 1 and the operations $\odot, \ominus, \rightarrow$ are as follows: $1=0^{*}, x \odot y=\left(x^{*} \oplus y^{*}\right)^{*}, x \ominus y=x \odot y^{*}$ and $x \rightarrow y=x^{*} \oplus y$ for any $x, y \in L$. We shall define $x \leq y$ if and only if $x^{*} \oplus y=1$, and check that $\leq$ is a partial order, called the natural order of $L$. The natural order determines a lattices structure, in which $x \vee y=\left(x \odot y^{*}\right) \oplus y$, $x \wedge y=x \odot\left(x^{*} \oplus y\right)$ for any $x, y \in L$. The structure $(L, \wedge, \vee, 0,1)$ is a bounded distributive lattice. We say that the MV-algebra $L$ is linearly ordered if the lattice $(L, \wedge, \vee, 0,1)$ is linearly ordered. An MV-algebra is a Boolean algebra if it satisfies the additional equation $x \oplus x=x$ (or $x \odot x=x$ ) for any $x \in L$, and denote by $B(L)=\{x \in L \mid x \oplus x=x\}$ be the set of all idempotent elements of $L$. As MV -algebras form an equational class, the notions of MVhomorphism, ideal, quotient, subalgebra, product, are just the particular cases of the corresponding universal algebraic notions $[3,5,6]$.

Let $(G,+, \leq)$ be an abelian $\ell$-group, i.e., an abelian group equipped with a compatible lattice ordered. For any $0 \leq u \in G$, the algebra $\Gamma(G, u)=$ $\left([0, u], \oplus_{u}, \neg, 0\right)$, where $x \oplus_{u} y=(x+y) \wedge u$ and $\neg x=u-x$, is an MValgebra. Up to isomorphism, all MV-algebras are of the form $\Gamma(G, u)$, where $u$ is a strong unit for $G$, i.e., the convex $\ell$-group of $G$ generated by $u$ is $G$. 
Even strongly, $\Gamma$ as a functor is an equivalence between the category of unital abelian $\ell$-groups and the category of MV-algebras [5].

Proposition 2.1 ([3, 4]). In an MV-algebra, the following properties hold: for any $x, y \in L$,

(1) $x \oplus x^{*}=1$,

(2) $x \ominus 0=x, 0 \ominus x=0, x \ominus x=0,1 \ominus x=x^{*}, x \ominus 1=0$,

(3) $x \leq y$ if and only if $x \rightarrow y=1$ if and only if $x \ominus y=0$,

(4) $x \odot y \leq x \wedge y$,

(5) $x \rightarrow(y \wedge z)=(x \rightarrow y) \wedge(x \rightarrow z)$,

(6) $x \rightarrow(y \vee z)=(x \rightarrow y) \vee(x \rightarrow z)$,

(7) if $x \leq y$, then $x \odot z \leq y \odot z, z \rightarrow x \leq z \rightarrow y, y \rightarrow z \leq x \rightarrow z$, $x \ominus z \leq y \ominus z, z \ominus y \leq z \ominus x$,

(8) $x \vee y=(x \rightarrow y) \rightarrow y=(y \rightarrow x) \rightarrow x$,

(9) $x \wedge y=y \ominus(y \ominus x)$,

(10) $x \odot(y \vee z)=(x \odot y) \vee(x \odot z)$,

(11) $x \oplus(y \wedge z)=(x \oplus y) \wedge(x \oplus z)$,

(12) $x \rightarrow(y \rightarrow z)=y \rightarrow(x \rightarrow z)$.

Proposition 2.2 ([3]). Let $L$ be an MV-algebra. Then the following statements are equivalent: for any $x, y \in L$,

(1) $x \in B(L)$,

(2) $x \oplus y=x \vee y$,

(3) $x \odot y=x \wedge y$.

Proposition 2.3 ([6]). Let $L$ be an MV-algebra and $e \in B(L)$. Then the following properties hold: for any $x, y \in L$,

(1) $e \wedge(x \odot y)=(e \wedge x) \odot(e \wedge y)$,

(2) $e \vee(x \odot y)=(e \vee x) \odot(e \vee y)$,

(3) $e \wedge(x \oplus y)=(e \wedge x) \oplus(e \wedge y)$,

(4) $e \vee(x \oplus y)=(e \vee x) \oplus(e \vee y)$,

(5) $e \odot(x \rightarrow y)=e \odot[(e \odot x) \rightarrow(e \odot y)]$

(6) $e \rightarrow(x \rightarrow y)=(e \rightarrow x) \rightarrow(e \rightarrow y)$.

Theorem 2.4 ([3]). (Chang's representation theorem) Every nontrivial $\mathrm{MV}$-algebra is a subdirect product of linearly ordered MV-algebras.

Galois connections play a central role in fuzzy logic systems and their algebraic semantics, hence we recall some well-known properties of orderpreserving Galois connections used here. Let $\mu: P \rightarrow Q$ and $\nu: Q \rightarrow P$ be two mappings between ordered sets $P$ and $Q$. The pair $(\mu, \nu)$ is a monotone Galois connection between $P$ and $Q$, if for all $p \in P$ and $q \in Q, \mu(p) \leq q$ if and only if $p \leq \nu(q)$. An equivalent characterization states a pair $(\mu, \nu)$ 
forms a Galois connection between $P$ and $Q$ if and only if it satisfying: (1) $p \leq \nu(\mu(q))$ for all $p \in P$ and $\mu(\nu(p)) \leq q$ for all $q \in Q,(2)$ the mappings $\mu$ and $\nu$ are order-preserving. Likewise by duality we can define the notion of antitone Galois connection [9].

\section{Implicative derivations on MV-algebras}

In this section, we investigate some basic properties of implicative derivations and give their characterizations in MV-algebras. Then, we show that every Boolean algebra is isomorphic to the algebra of all implicative derivations. Finally, we obtain that a direct product of MV-algebras by regular implicative derivations.

Implicative derivations were first introduced by $\mathrm{He}$ in residtaed lattices [11], we applied the notion of that to MV-algebras as follows.

Definition 3.1. Let $L$ be an MV-algebra. A mapping $g: L \longrightarrow L$ is called an implicative derivation on $L$ if it satisfies the following condition: for any $x, y \in L$,

$$
g(x \rightarrow y)=(g(x) \rightarrow y) \vee(x \rightarrow g(y))
$$
$I(L)$.

We will denote the set of all implicative derivations of an MV-algebra by

Example 3.2. Let $L$ be an MV-algebra. Define a mapping $1_{g}: L \longrightarrow L$ by $1_{g}(x)=1$ for all $x \in L$, then $1_{g}$ is an implicative derivation on $L$. Moreover, we define a mapping $g_{1}: L \longrightarrow L$ by $g_{1}(x)=x$ for all $x \in L$. Then $g_{1}$ is an implicative derivation on $L$, which is called an identity implicative derivation.

Example 3.3. Let $L$ be an MV-algebra and $a \in L$. Define a mapping $g_{a}$ : $L \longrightarrow L$ by $g_{a}(x)=a \rightarrow x$ for all $x \in L$, then $g_{a}$ is an implicative derivation on $L$, which is called a simple implicative derivation.

Example 3.4. Let $L=[0,1]$ be the real unit interval. If we define $x \oplus y=$ $\min \{1, x+y\}$ and $x^{*}=1-x$ for any $x, y \in L$, then $(L, \oplus, *, 0)$ is an MV-algebra, which is called a standard Eukasiewicz structure. Also, for each number $n \geq 2$, the $n$-element set $S_{n}=\left\{0, \frac{1}{n-1}, \frac{2}{n-1}, \cdots, \frac{n-1}{n-1}, 1\right\}$ is a subalgebra of $L$. Now, we define a mapping $g: S_{n} \rightarrow S_{n}$ as follows: for all $x \in S_{n}$,

$$
g(x)= \begin{cases}\frac{1}{n-1}, & x=0 \\ \frac{1}{n-1} \oplus x, & x \neq 0\end{cases}
$$

It is verified that $g$ is an implicative derivation on $S_{n}$. 
Proposition 3.5. Let $g$ be an implicative derivation on $L$. Then the following properties hold: for any $x, y \in L$,

(1) $g(1)=1$,

(2) $x \leq g(x)$,

(3) $g(x) \rightarrow y \leq x \rightarrow g(y)$,

(4) if $x \leq y$, then $g(x) \leq g(y)$,

(5) $g(x)=x \oplus g(0)$,

(6) $g(x \wedge y)=g(x) \wedge g(y)$,

(7) $g(x \oplus y) \leq g(x) \oplus g(y)$.

Proof. For (1)-(3) see ([11], Proposition 4.8), we only to prove (4)-(7).

(4) If $x \leq y$, then follows from Proposition 2.1(8) that

$$
\begin{aligned}
g(y) & =g(x \vee y)=g((y \rightarrow x) \rightarrow x) \\
& =(g(y \rightarrow x) \rightarrow x) \vee((y \rightarrow x) \rightarrow g(x)) \\
& \geq(y \rightarrow x) \rightarrow g(x) \\
& \geq g(x) .
\end{aligned}
$$

Thus, $g(x) \leq g(y)$.

(5) For any $x \in L$, by Definition 3.1, we have

$$
\begin{aligned}
g(x) & =g\left(x^{* *}\right)=g\left(x^{*} \rightarrow 0\right) \\
& =\left(g\left(x^{*}\right) \rightarrow 0\right) \vee\left(x^{*} \rightarrow g(0)\right) \\
& =\left(g\left(x^{*}\right)\right)^{*} \vee(x \oplus g(0)) .
\end{aligned}
$$

Also, by (2), we have $\left(g\left(x^{*}\right)\right)^{*} \leq x^{* *}=x$, and so $g(x)=x \oplus g(0)$ for any $x \in L$.

(6) For any $x, y \in L$, by (5) and Proposition 2.1(11), we have

$$
\begin{aligned}
g(x \wedge y) & =g(0) \oplus(x \wedge y) \\
& =(g(0) \oplus x) \wedge(g(0) \oplus y) \\
& =g(x) \wedge g(y) .
\end{aligned}
$$

Thus, $g(x \wedge y)=g(x) \wedge g(y)$ for any $x, y \in L$.

(7) For any $x, y \in L$, from (5), we have

$$
\begin{aligned}
g(x \oplus y) & =g(0) \oplus(x \oplus y) \\
& \leq g(0) \oplus g(0) \oplus(x \oplus y) \\
& =(g(0) \oplus x) \oplus(g(0) \oplus y) \\
& =g(x) \oplus g(y) .
\end{aligned}
$$

Thus, $g(x \oplus y) \leq g(x) \oplus g(y)$ for any $x, y \in L$. 
Remark 3.6. (1) Proposition 3.5(3) gives us an ideal of introducing an implicative derivation on an MV-algebra in a different way. Namely, we can consider a mapping $g: L \rightarrow L$ satisfying

$$
g(x \rightarrow y)=x \rightarrow g(y)
$$

for any $x, y \in L$. As an application of the above statement, we can obtain that $g_{a}$ in Example 3.2 is an implicative derivation, since

$$
g_{a}(x \rightarrow y)=a \rightarrow(x \rightarrow y)=x \rightarrow(a \rightarrow y)=x \rightarrow g_{a}(y)
$$

for any $x, y \in L$.

(2) Proposition 3.5(5) shows that every implicative derivation $g$ is completely determined by the element $g(0)$, however, it does not hold in residuated lattices in general (see [11], Example 4.3). Also, we can prove that a residuated lattice is regular, which is a residuated lattice satisfies $x^{* *}=x$, if and only if it satisfies Proposition 3.5(5). As a corollary of the above statement, every BL-algebra is an MV-algebra if and only if it satisfies Proposition 3.5(5), since BL-algebra is a subclass of residuated lattice based on continuous t-norm and its residua. In this case, $x^{* *}=x$ is equivalent to $(x \rightarrow y) \rightarrow y=(y \rightarrow x) \rightarrow x$ for any $x, y \in L$.

By means of implicative derivation, we give some characterizations for an MV-algebra to be a Boolean algebra.

Theorem 3.7. Let $L$ be an MV-algebra. Then the following statements are equivalent: for any $x, y \in L$,

(1) $L$ is a Boolean algebra,

(2) every implicative derivation $g$ on $L$ satisfies $g(x \wedge y)=g(x) \odot g(y)$,

(3) every implicative derivation $g$ on $L$ satisfies $g(x \vee y)=g(x) \oplus g(y)$.

Proof. (1) $\Rightarrow(2)$ If $L$ is a Boolean algebra, then $L$ satisfies $x \odot y=x \wedge y$ for any $x, y \in L$. For any $x, y \in L$, by Proposition 3.5(6), we have

$$
g(x \wedge y)=g(x) \wedge g(y)=g(x) \odot g(y) .
$$

Thus, $g(x \wedge y)=g(x) \odot g(y)$ for any $x, y \in L$.

$(2) \Rightarrow(1)$ From Example 3.2, we know that $g_{1}$ is an implicative derivation on $L$. Taking $g=g_{1}$, we have $x \odot y=x \wedge y$ for all $x, y \in L$, which implies that $L$ is a Boolean algebra.

$(1) \Rightarrow(3)$ If $L$ is a Boolean algebra, then $L$ satisfies $x \oplus y=x \vee y$ for any $x, y \in L$. For any $x, y \in L$, from Proposition 3.5(7), we have

$$
g(x) \vee g(y) \leq g(x \vee y)=g(x \oplus y) \leq g(x) \oplus g(y)=g(x) \vee g(y) .
$$

Thus, $g(x \vee y)=g(x) \oplus g(y)$ for any $x, y \in L$.

$(3) \Rightarrow(1)$ The proof is similar to that of $(2) \Rightarrow(1)$. 
Also, we give some characterizations of implicative derivations on MValgebras.

Theorem 3.8. Let $g$ be a self mapping on $L$. Then the following statements are equivalent: for any $x, y \in L$,

(1) $g$ is an implicative derivation on $L$,

(2) $g(x)=x \oplus g(0)$,

(3) $g(x \oplus y)=x \oplus g(y)$,

(4) $g(x \rightarrow y)=x \rightarrow g(y)$.

Proof. $(1) \Rightarrow(2)$ It follows from Proposition 3.5(5).

$(2) \Rightarrow(3)$ For any $x, y \in L$, by (2), we have

$$
g(x \oplus y)=(x \oplus y) \oplus g(0)=x \oplus(y \oplus g(0))=x \oplus g(y) .
$$

Thus, $g(x \oplus y)=x \oplus g(y)$ for any $x, y \in L$.

$(3) \Rightarrow(4)$ For any $x, y \in L$, by (3), we have

$$
g(x \rightarrow y)=g\left(x^{*} \oplus y\right)=x^{*} \oplus g(y)=x \rightarrow g(y) .
$$

Thus, $g(x \rightarrow y)=x \rightarrow g(y)$ for any $x, y \in L$.

(4) $\Rightarrow$ (1) It follows from Remark 3.6(1).

The following theorems give a representation of Boolean algebras in terms of implicative derivations. Namely, every Boolean algebra is isomorphic to the algebra of all implicative derivations.

Theorem 3.9. Let $L$ be an MV-algebra. Then $\left(I(L), \cap, \cup, g_{1}, 1_{g}\right)$ is a bounded distributive lattice, where $\left(g_{i} \cap g_{j}\right)(x)=g_{i}(x) \wedge g_{j}(x),\left(g_{i} \cup g_{j}\right)(x)=g_{i}(x) \vee$ $g_{j}(x), g_{1}(x)=x, 1_{g}(x)=1$, for any $g_{i}, g_{j} \in I(L), x \in L$.

Proof. For any $g_{i}, g_{j} \in I(L)$ and $x \in L$, by Propositions 2.1(5) and Theorem $3.8(4)$, we have

$$
\begin{aligned}
\left(g_{i} \cap g_{j}\right)(x \rightarrow y) & =\left(g_{i}(x \rightarrow y)\right) \wedge\left(g_{j}(x \rightarrow y)\right) \\
& =\left(x \rightarrow g_{i}(y)\right) \wedge\left(x \rightarrow g_{j}(y)\right) \\
& =x \rightarrow\left(g_{i}(y) \wedge g_{j}(y)\right) \\
& =x \rightarrow\left(g_{i} \cap g_{j}\right)(y),
\end{aligned}
$$

which implies $g_{i} \cap g_{j} \in I(L)$.

Also, it follows from Proposition 2.1(6) and Theorem 3.8(4) that

$$
\begin{aligned}
\left(g_{i} \cup g_{j}\right)(x \rightarrow y) & =\left(g_{i}(x \rightarrow y)\right) \vee\left(g_{j}(x \rightarrow y)\right) \\
& =\left(x \rightarrow g_{i}(y)\right) \vee\left(x \rightarrow g_{j}(y)\right) \\
& =x \rightarrow\left(g_{i}(y) \vee g_{j}(y)\right) \\
& =x \rightarrow\left(g_{i} \cup g_{j}\right)(y),
\end{aligned}
$$


which implies $g_{i} \cup g_{j} \in I(L)$.

Moreover, for any $g_{i} \in I(L), x \in L$, we have

$$
\left(g_{i} \cap g_{1}\right)(x)=g_{i}(x) \wedge g_{1}(x)=g_{i}(x) \wedge x=x=g_{1}(x)
$$

and

$$
\left(g_{i} \cup 1_{g}\right)(x)=g_{1}(x) \vee 1_{g}(x)=g_{i}(x) \vee 1=1=1_{g}(x),
$$

which implies that $g_{1}$ is the smallest element and $1_{g}$ is the greatest element of $I(L)$.

Thus, $\left(I(L), \cap, \cup, g_{1}, 1_{g}\right)$ is a bounded lattice. Since $(L, \wedge, \vee, 0,1)$ is a bounded distributive lattice, $\left(I(L), \cap, \cup, g_{1}, 1_{g}\right)$ is also a bounded distributive lattice.

Theorem 3.10. Let $(L, \wedge, \vee, *, 0,1)$ be a Boolean algebra. Then $\left(I(L), \cap, \cup, \neg, g_{1}, 1_{g}\right)$ is a Boolean algebra, where $\left(g_{i} \cap g_{j}\right)(x)=\left(g_{i}(x)\right) \wedge\left(g_{j}(x)\right),\left(g_{i} \cup g_{j}\right)(x)=$ $\left(g_{i}(x)\right) \vee\left(g_{j}(x)\right),\left(g_{i} \Rightarrow g_{j}\right)(x)=g_{i}(x) \rightarrow g_{j}(x), \neg\left(g_{i}\right)(x)=\left(g_{i}(x)\right) \rightarrow\left(g_{1}(x)\right)$, $g_{1}(x)=x, 1_{g}(x)=1$, for any $g_{i}, g_{j} \in I(L), x \in L$.

Proof. Theorem 3.9 shows that $\left(I(L), \cap, \cup, g_{1}, 1_{g}\right)$ is a bounded distributive lattice. Now, we prove that $\left(I(L), \cap, \cup, \neg, g_{1}, 1_{g}\right)$ is a Boolean algebra if $L$ is a Boolean algebra. Indeed, for any $g_{i}, g_{j} \in I(L)$ and $x \in L$, by Proposition 2.3(6) and Theorem 3.8(4), we have

$$
\begin{aligned}
\left(g_{i} \Rightarrow g_{j}\right)(x \rightarrow y) & =g_{i}(x \rightarrow y) \rightarrow g_{j}(x \rightarrow y) \\
& =\left(x \rightarrow g_{i}(y)\right) \rightarrow\left(x \rightarrow g_{j}(y)\right) \\
& =x \rightarrow\left(g_{i}(y) \rightarrow g_{j}(y)\right) \\
& =x \rightarrow\left(g_{i} \Rightarrow g_{j}\right)(y),
\end{aligned}
$$

which implies $g_{i} \Rightarrow g_{j} \in I(L)$.

Moreover, we have

$$
\begin{aligned}
\left(g_{i} \cap \neg g_{i}\right)(x) & =g_{i}(x) \wedge \neg g_{i}(x) \\
& =g_{i}(x) \wedge\left(g_{i}(x) \rightarrow g_{1}(x)\right) \\
& =g_{i}(x) \wedge g_{1}(x) \\
& =g_{1}(x), \\
\left(g_{i} \cup \neg g_{i}\right)(x) & =g_{i}(x) \vee\left(g_{i}(x) \rightarrow g_{1}(x)\right) \\
& =g_{i}(x) \vee\left(\left(g_{i}(x)\right)^{*} \vee g_{1}(x)\right) \\
& =\left(g_{i}(x) \vee\left(g_{i}(x)\right)^{*}\right) \vee g_{1}(x) \\
& =1 \vee g_{1}(x) \\
& =1 \\
& =1_{g}(x),
\end{aligned}
$$


which implies $g_{i} \cap \neg g_{i}=g_{1}$ and $g_{i} \cup \neg g_{i}=1_{g}$.

Thus $\left(I(L), \cap, \cup, \neg, g_{1}, 1_{g}\right)$ is a Boolean algebra.

We have seen Theorem 3.29 in [20] that every distributive lattice is isomorphic to the algebra of all principal derivations. The corresponding result for Boolean algebra is as follows.

Theorem 3.11. Let $(L, \wedge, \vee, *, 0,1)$ be a Boolean algebra. Then $(L, \wedge, \vee, *, 0,1)$ is isomorphic to $\left(I(L), \cap, \cup, \neg, g_{1}, 1_{g}\right)$.

Proof. Let $\phi: L \longrightarrow I(L)$ be defined by $\phi(a)(x)=a \vee x$, for all $a, x \in L$. Then follows from Theorem 3.8(2) that $\phi$ is well defined.

(1) If $\phi(a)=\phi(b)$, then $\phi(a)(x)=\phi(b)(x)$, and so $a \vee x=b \vee x$ for all $x \in L$. Now, if $x=a$, then $a \vee a=b \vee a$, so $b \vee a=a$, that is, $b \leq a$. If $x=b$, then $a \vee b=b \vee b$, and hence $a \vee b=b$, that is, $a \leq b$. So $a=b$, which implies that $\phi$ is an injective mapping.

(2) For any $g \in I(L)$, there exists $g(0) \in L$ such that $g=\phi((g(0)))$, which implies that $\phi$ is a surjection mapping. Indeed, by Theorem $3.8(1) \Rightarrow(2)$, we have $g(x)=g(0) \oplus x=g(0) \vee x=\phi(g(0))(x)$ for any $x \in L$.

(3) For any $a, b, x \in L$, we have

$$
\begin{aligned}
\phi(a \wedge b)(x) & =(a \wedge b) \vee x=(a \vee x) \wedge(b \vee x)=(\phi(a) \cap \phi(b))(x), \\
\phi(a \vee b)(x) & =(a \vee b) \vee x=(a \vee x) \vee(b \vee x)=(\phi(a) \cup \phi(b))(x), \\
\phi\left(a^{*}\right) & =a^{*} \vee x \\
& =a \rightarrow x \\
& =(a \rightarrow x) \wedge(x \rightarrow x) \\
& =(a \vee x) \rightarrow x \\
& =\phi(a)(x) \rightarrow g_{1}(x) \\
& =\neg \phi(a)(x),
\end{aligned}
$$

which implies that $\phi$ is a homomorphism.

Therefore $(L, \wedge, \vee, *, 0,1)$ and $\left(I(L), \cap, \cup, \neg, g_{1}, 1_{g}\right)$ are isomorphic.

In order to obtain a representation of MV-algebras, we introduce the regular implicative derivation as following.

Definition 3.12. An implicative derivation $g$ is said to be regular if $g(0) \in$ $B(L)$.

Remark 3.13. (1) Examples 3.3 and 3.4 show that implicative derivations are not regular implicative derivations, in general.

(2) The implicative derivation in Example 3.3 is regular if and only if $a \in B(L)$. Indeed, if $a \in B(L)$, then $g_{a}(0)=a \rightarrow 0=a^{*} \in B(L)$. Conversely, 
if $g_{a}$ is regular, then $g_{a}(0)=g_{a}(0) \oplus g_{a}(0)$, which implies $a^{*}=a^{*} \oplus a^{*}$, and so $a \in B(L)$.

(3) There is no nontrivial regular implicative derivation on 2-valued Boolean algebra. Indeed, it is easily verified that $B(L)=\{0,1\}$ if and only if either regular implicative derivation $d$ is an identity derivation or a zero derivation.

Proposition 3.14. Let $g$ be a regular implicative derivation on $L$. Then the following hold: for any $x, y \in L$,

(1) $g(g(x))=g(x)$,

(2) $g(x \oplus y)=g(x) \oplus g(y)$,

(3) $g(L)=$ Fix $_{g}(L)$, where Fix $(L)=\{x \in L \mid g(x)=x\}$.

Proof. (1) For any $x \in L$, by Proposition 3.5(5), we have

$$
\begin{aligned}
g(g(x)) & =g(x \oplus g(0)) \\
& =x \oplus g(0) \oplus g(0) \\
& =x \oplus g(0) \\
& =g(x) .
\end{aligned}
$$

Thus, $g(g(x))=g(x)$ for any $x \in L$.

(2) For any $x, y \in L$, by Proposition 3.5(5), we have

$$
\begin{aligned}
g(x \oplus y) & =g(0) \oplus(x \oplus y) \\
& =g(0) \oplus g(0) \oplus x \oplus y \\
& =(g(0) \oplus x) \oplus(g(0) \oplus y) \\
& =g(x) \oplus g(y) .
\end{aligned}
$$

Thus, $g(x \oplus y)=g(x) \oplus g(y)$ for any $x, y \in L$.

(3) It follows from (1).

Proposition 3.15. Let $g$ be a regular implicative derivation on $L$. Then $(g(L), \oplus, \star, g(0))$ is also an MV-algebra, where $x^{\star}=g\left(x^{*}\right)$ for any $x \in g(L)$.

Proof. It follows from Propositions 3.5 and 3.14.

The following theorem gives a representation of MV-algebras in terms of regular implicative derivations.

Theorem 3.16. Every $\mathrm{MV}$-algebra $L$ is isomorphic to the direct product $\left(g(L), \oplus, \star_{1}, g(0)\right)$ and $\left(g_{*}(L), \oplus, \star_{2},(g(0))^{*}\right)$, where $g_{*}(L)=\left\{x \in L \mid g^{*}(x)=\right.$ $x\}, g^{*}(x)=g(0) \rightarrow x$ and $g(0) \in B(L)$. 
Proof. Let $\varphi: L \rightarrow g(L) \times g_{*}(L)$ be defined by $\varphi(x)=\left(g(x), g^{*}(x)\right)$ for all $x \in L$. Then follows from Propositions 3.14 and 3.15 that $\varphi$ is well defined and a surjection homomorphism from $L$ to $g(L) \times g_{*}(L)$.

Also, if $x_{1} \in g(L)$ and $x_{2} \in g_{*}(L)$, for $x=x_{1} \wedge x_{2}$, then $\varphi(x)=\left(x_{1}, x_{2}\right)$. Since $(L, \wedge, \vee, 0,1)$ is a distributive lattice, we have

$$
x=(g(0) \vee x) \wedge\left((g(0))^{*} \vee x\right)=(g(0) \oplus x) \wedge(g(0) \rightarrow x)=g(x) \wedge g^{*}(x)
$$

for all $x \in L$, which implies that $\varphi$ is a injective mapping.

It is easy to verify that $\varphi$ is bijection and that $\varphi^{-1}(x, y)=x \wedge y$, for all $(x, y) \in g(L) \times g_{*}(L)$, is also a surjection homomorphism from $g(L) \times g_{*}(L)$ to $L$.

\section{Difference derivations on MV-algebras}

In this section, we give some characterizations of difference derivations and discuss the relationship between the regular derivation pair and the Galois connection on MV-algebras. Also, we prove that regular difference derivations coincide with direct product decompositions of MV-algebras.

Definition 4.1. Let $L$ be an MV-algebra. A mapping $d: L \longrightarrow L$ is called a difference derivation on $L$ if it satisfies the following condition: for any $x, y \in L$,

$$
d(x \ominus y)=(d(x) \ominus y) \wedge(x \ominus d(y))
$$

We will denote the set of all difference derivations of an MV-algebra by $D(L)$.

Example 4.2. Let $L$ be an MV-algebra. Define a mapping $d_{0}: L \longrightarrow L$ by $d_{0}(x)=0$ for all $x \in L$, then $d_{0}$ is a difference derivation on $L$, which is called a zero difference derivation. Moreover, we define a mapping $d_{1}: L \longrightarrow L$ by $d_{1}(x)=x$ for all $x \in L$. Then $d$ is a difference derivation on $L$, which is called an identity difference derivation.

Example 4.3. Let $L$ be an MV-algebra and $a \in L$. Define a mapping $d_{a}$ : $L \longrightarrow L$ by $d_{a}(x)=x \odot a$ for all $x \in L$, then $d_{a}$ is a difference derivation on $L$, which is called a simple difference derivation.

Example 4.4. Considering MV-algebra $S_{n}$ in Example 3.4. Now, we define a mapping $d: S_{n} \rightarrow S_{n}$ as follows: for all $x \in S_{n}$,

$$
d(x)= \begin{cases}\frac{1}{n-1}, & x=1 \\ \frac{1}{n-1} \odot x, & x \neq 1\end{cases}
$$


It is easily verified that $d$ is a difference derivation on $S_{n}$.

Proposition 4.5. Let $d$ be a difference derivation on $L$. Then the following hold: for any $x, y \in L$,

(1) $d(0)=0$,

(2) $d(x) \leq x$,

(3) if $x \leq y$, then $d(x) \leq d(y)$,

(4) $d(x) \ominus y \leq x \ominus d(y)$,

(5) $d(x)=d(1) \odot x$

(6) $d(x) \odot y=x \odot d(y)$,

(7) $d(x \wedge y)=d(x) \wedge d(y)$,

(8) $d(x \vee y)=d(x) \vee d(y)$.

Proof. The proofs of (4),(6)-(8) are easy, we only to prove (1)-(3),(5).

(1) From Proposition 2.1(2), we have

$$
d(0)=d(0 \ominus 0)=(d(0) \ominus 0) \wedge(0 \ominus d(0))=0 .
$$

(2) For any $x \in L$, by Proposition 2.1(2), we have

$$
d(x)=d(x \ominus 0)=(d(x) \ominus 0) \wedge(x \ominus d(0))=d(x) \wedge x .
$$

Thus, $d(x) \leq x$ for any $x \in L$.

(3) If $x \leq y$, then $x=x \wedge y=y \ominus(y \ominus x)$, and hence

$$
\begin{aligned}
d(x) & =d(y \ominus(y \ominus x)) \\
& =(d(y) \ominus(y \ominus x)) \wedge(y \ominus d(y \ominus x)) \\
& \leq d(y) \ominus(y \ominus x) \\
& \leq d(y),
\end{aligned}
$$

and so $d(x) \leq d(y)$.

(5) For any $x \in L$, by Proposition 2.1(2), we have

$$
d(x)=d\left(1 \ominus x^{*}\right)=\left(d(1) \ominus x^{*}\right) \wedge\left(1 \ominus d\left(x^{*}\right)\right)=(d(1) \odot x) \wedge\left(d\left(x^{*}\right)\right)^{*} .
$$

Also, from (2), we have $x=x^{* *} \leq\left(d\left(x^{*}\right)\right)^{*}$. Thus, $d(x)=d(1) \odot x$ for any $x \in L$.

Remark 4.6. Proposition 4.5(4) gives us an ideal of introducing a difference derivation on an MV-algebra in a different way. Namely, we can consider a mapping $d: L \rightarrow L$ satisfying

$$
d(x \ominus y)=d(x) \ominus y
$$


for any $x, y \in L$. As an application of the above statement, we have that $d_{a}$ in Example 4.3 is a difference derivation, since

$$
d_{a}(x \ominus y)=a \odot(x \ominus y)=a \odot\left(x \odot y^{*}\right)=(a \odot x) \odot y^{*}=d_{a}(x) \ominus y
$$

for any $x, y \in L$.

Next, we give some characterizations of difference derivations on MValgebras.

Theorem 4.7. Let $d$ be a self mapping on $L$. Then the following statements are equivalent: for any $x, y \in L$,

(1) $d$ is a difference derivation on $L$,

(2) $d(x)=d(1) \odot x$,

(3) $d(x \odot y)=d(x) \odot y$,

(4) $d(x \ominus y)=d(x) \ominus y$.

Proof. $(1) \Rightarrow(2)$ It follows from Proposition 4.5(5).

$(2) \Rightarrow(3)$ For any $x, y \in L$, by Proposition $4.5(5)$, we have

$$
d(x \odot y)=d(1) \odot(x \odot y)=x \odot(d(1) \odot y)=x \odot d(y) .
$$

$(3) \Rightarrow(4)$ For any $x, y \in L$, by (3), we have

$$
d(x \ominus y)=d\left(x \odot y^{*}\right)=d(x) \odot y^{*}=d(x) \ominus y .
$$

$(4) \Rightarrow(1)$ It follows from Remark 4.6.

Let $\alpha: D(L) \longrightarrow I(L)$ be the mapping such that $\alpha(d)(x)=\left(d\left(x^{*}\right)\right)^{*}$ for any $d \in D(L), x \in L$, and $\beta: I(L) \longrightarrow D(L)$ be the mapping such that $\beta(g)(x)=\left(g\left(x^{*}\right)\right)^{*}$ for any $g \in I(L), x \in L$.

Theorem 4.8. The $\alpha$ and $\beta$ form an antitone Galois connection between $D(L)$ and $I(L)$. Namely, $d \leq \beta(g)$ if and only if $g \leq \alpha(d)$ for any $d \in D(L)$ and $g \in I(L)$.

Proof. It follows from Propositions 4.5(3) and 3.5(4) that $\alpha$ and $\beta$ are antitone.

If $d \leq \beta(g)$, then $d(x) \leq \beta(g)(x)=\left(g\left(x^{*}\right)\right)^{*}$, and hence $(d(x))^{*} \geq g\left(x^{*}\right)$ for any $x \in L$. So $\left(d\left(x^{*}\right)\right)^{*} \geq g(x)$, which implies $\alpha(d)(x) \geq g(x)$ for any $x \in L$. Thus $g \leq \alpha(d)$.

Conversely, if $g \leq \alpha(d)$, then $g(x) \leq \alpha(d)(x)=\left(d\left(x^{*}\right)\right)^{*}$, and hence $d\left(x^{*}\right) \leq$ $(g(x))^{*}$, which implies $d(x) \leq\left(g\left(x^{*}\right)\right)^{*}=\beta(g)(x)$ for any $x \in L$. Thus $d \leq$ $\beta(g)$.

Definition 4.9. A difference derivation $d$ is said to be regular if $d(1) \in B(L)$. 
Remark 4.10. (1) Examples 4.3 and 4.4 show that difference derivations are not regular, in general.

(2) The difference derivation $d_{a}$ in Example 4.4 is regular if and only if $a \in B(L)$. The proof is similar to that of Remark 3.13(2).

Proposition 4.11. Let $d$ be a regular difference derivation on $L$. Then the following hold: for any $x, y \in L$,

(1) $d(d(x))=d(x)$,

(2) $d(x \odot y)=d(x) \odot d(y)$,

(3) $d(x \oplus y)=d(x) \oplus d(y)$,

(4) $d(L)=$ Fix $_{d}(L)$, where Fix $(L)=\{x \in L \mid d(x)=x\}$.

Proof. The proof is similar to that of Proposition 3.14.

Let $L$ be an MV-algebra and $g_{d}, d_{g}: L \rightarrow L$ be the mappings such that $g_{d}(x)=\left(d\left(x^{*}\right)\right)^{*}$, and $d_{g}(x)=\left(g\left(x^{*}\right)\right)^{*}$ for any $x \in L, d \in D(L), g \in I(L)$.

Theorem 4.12. There exists a one to one correspondence between $I(L)$ and $D(L)$. Namely,

(1) $g_{d}$ is a regular implicative derivation on $L$,

(2) $d_{g}$ is a regular difference derivation on $L$,

(3) $d_{\left(g_{d}\right)}=d$ and $g_{\left(d_{g}\right)}=g$.

Proof. If $d$ is a regular difference derivation on $L$, then

$$
\begin{aligned}
g_{d}(x \rightarrow y) & =\left(d(x \rightarrow y)^{*}\right)^{*}=\left(d\left(x^{*} \oplus y\right)^{*}\right)^{*} \\
& =\left(d\left(x \odot y^{*}\right)\right)^{*}=\left(x \odot d\left(y^{*}\right)\right)^{*} \\
& =x^{*} \oplus\left(d\left(y^{*}\right)\right)^{*} \\
& =x \rightarrow g_{d}(y),
\end{aligned}
$$

for any $x, y \in L$, which implies that $g_{d}$ is an implicative derivation on $L$. Also, since $d(1) \in B(L)$, we have $g_{d}(0)=\left(d\left(0^{*}\right)\right)^{*}=(d(1))^{*} \in B(L)$. Thus $g_{d}$ is regular.

Conversely, if $g$ is a regular implicative derivation on $L$, then

$$
\begin{aligned}
d_{g}(x \odot y) & =\left(g\left((x \odot y)^{*}\right)\right)^{*}=\left(g\left(x^{*} \oplus y^{*}\right)\right)^{*} \\
& =\left(g\left(x \rightarrow y^{*}\right)\right)^{*}=\left(x \rightarrow g\left(y^{*}\right)\right)^{*} \\
& =x \odot\left(g\left(y^{*}\right)\right)^{*} \\
& =x \odot d_{g}(y),
\end{aligned}
$$

for any $x, y \in L$, which implies that $d_{g}$ is a difference derivation on $L$. Also, since $g(0) \in B(L)$, we have $d_{g}(1)=\left(g\left(1^{*}\right)\right)^{*}=(g(0))^{*} \in B(L)$. Thus $d_{g}$ is regular. 
Moreover, we have

$$
\begin{aligned}
d_{\left(g_{d}\right)}(x) & =d\left(\left(d\left(x^{*}\right)\right)^{*}\right)=d(1) \odot\left((d(1))^{*} \oplus x\right) \\
& =\left(d(1) \odot(d(1))^{*}\right) \oplus(d(1) \odot x) \\
& =d(1) \odot x \\
& =d(x)
\end{aligned}
$$

for any $x \in L$, and so $d_{\left(g_{d}\right)}=d$. Similarity, $g_{\left(d_{g}\right)}=g$.

We denote the pair of regular difference and implicative derivations of an MV-algebra $L$ by $(d, g)$, which is called the regular derivation pair on $L$.

In what follows, we discuss the relationship between the regular derivation pair and the Galois connection on MV-algebra $L$.

Proposition 4.13. Let $(d, g)$ be a regular derivation pair on $L$. Then $(d, g)$ establishes a Galois connection between $d(L)$ and $g(L)$.

Proof. (1) For all $x \in g(L) \subseteq L$, by Proposition 4.11(1), we have $d(x) \in d(L)$, and hence $d$ is a well defined mapping from $g(L)$ to $d(L)$. Similarity, $g$ is a well defined mapping from $d(L)$ to $g(L)$.

(2) Applying Propositions 3.5(4) and 4.5(3), we obtain that $g$ and $d$ are all isotone mappings between $d(L)$ and $g(L)$.

(3) If $y \leq d(x)(g(y) \leq x)$, then follows from Propositions 4.5(2)(3.5(2)) that $d(y) \leq d(x)(g(y) \leq g(x))$, and hence $g(y) \leq x(y \leq d(x))$ for any $x, y \in L$.

Thus $(d, g)$ establishes a Galois connection between $d(L)$ and $g(L)$.

The following example shows that the regular derivation pair does not forms a Galois connection on $L$, in general.

Example 4.14. Considering MV-algebra $S_{3}$ in Example 3.4. From Examples 3.2 and 4.1 , we know that $1_{g}$ and $d_{0}$ are regular implicative and difference derivation on $L$, respectively. However, $\left(1_{g}, d_{0}\right)$ does not forms a Galois connection on $S_{3}$, since $d_{0}\left(\frac{1}{2}\right)=0 \geq 0, g_{1}(0)=1 \not \frac{1}{2}$.

In what follows, we prove that every regular derivation pair is a Galois connection on $L$ if and only if $d(L)=g(L)$.

Theorem 4.15. Let $(d, g)$ be a regular derivation pair on $L$. Then the following statements are equivalent:

(1) $(d, g)$ forms a Galois connection on $L$,

(2) $d(L)=g(L)$. 
Proof. $(1) \Rightarrow(2)$ If $(d, g)$ establishes a Galois connection on $L$, then $g(x)=$ $g d(x) \leq x$, further by Proposition 3.5(2), we have $g(x)=x$ for any $x \in d(L)$. Thus $d(L) \subseteq g(L)$. Similarity, $g(L) \subseteq d(L)$. So $d(L)=g(L)$.

$(2) \Rightarrow(1)$ If $y \leq d(x)$, then $g d(x)=g(d(x)) \geq g(y)$ further by $d(x) \in$ $d(L)=g(L)$, we have $g d(x)=g(d(x))=d(x)$, and hence $g(y) \leq d(x) \leq x$ for any $x, y \in L$. Thus $g(y) \leq x$. Similarity, $g(y) \leq x$ implies $y \leq d(x)$. Then follows from Propositions 3.5(4) and 4.5(3) that $d$ and $g$ are isotone. Thus $(d, g)$ establishes a Galois connection on $L$.

Example 4.16. Considering two mappings $d_{a}(x)=a \odot x, g_{a}(x)=a \rightarrow x$ for any $a \in L / B(L), x \in L$, on MV-algebra $L$. It is easy to check that $\left(d_{a}, g_{a}\right)$ forms a Galois connection on $L$, but it does not form a regular derivation pair on $L$.

Inspired by Theorem 4.15 and Example 4.16, we have the following problem.

Open problem. Under which suitable conditions, every Galois connection is a regular derivation pair on MV-algebra $L$ ?

Our objective now is to obtain the relationship between regular difference derivations and the direct product decompositions of MV-algebras. For this purpose, we require the following facts.

Proposition 4.17. Let $d$ be a regular implicative derivation on $L$. Then $d(L)$,

(1) $\left(d(L), \oplus, \star_{3}, d(0)\right)$ is an $M V$-algebra, where $x^{\star}=d\left(x^{*}\right)$ for any $x \in$

(2) $d: L \longrightarrow d(L)$ is a surjective homomorphism,

(3) $\bar{d}: L / K e r(d) \longrightarrow d(L)$ is an isomorphism.

Proof. It follows from Proposition 4.11.

Theorem 4.18. Every MV-algebra $L$ is isomorphic to the direct product $\left(d(L), \oplus, \star_{3}, d(0)\right)$ and $\left(d_{*}(L), \oplus, \star_{4},(d(0))^{*}\right)$, where $d_{*}(L)=\left\{x \in L \mid d^{*}(x)=\right.$ $x\}, d^{*}(x)=(d(1))^{*} \odot x$ and $d(1) \in B(L)$.

Proof. The proof is similar to that of Theorem 3.16.

Corollary 4.19. Every MV-algebra $L$ is isomorphic to the direct product $\left([d(0), 1], \oplus, \star_{3^{\prime}}, d(0)\right)$ and $\left(\left[(d(0))^{*}, 1\right], \oplus, \star_{4^{\prime}},(d(0))^{*}\right)$.

Proof. It follows from Theorems 2.4 and 4.18.

The following theorem shows that there exists a one to one correspondence between regular implicative derivations and direct product decompositions on MV-algebras. 
Theorem 4.20. Let $L$ be an MV-algebra. Then there exists a one to one correspondence between the regular difference derivations on $L$ and the direct product decompositions $L \cong L_{1} \times L_{2}$, where $L_{1}=\left([e, 1], \oplus, \star_{3^{\prime}}, e\right), L_{2}=$ $\left(\left[e^{*}, 1\right], \oplus, \star_{4^{\prime}}, e^{*}\right)$.

Proof. Now, let $d$ be a regular difference derivation on MV-algebra such that $e=d(1)$. Further, let $L_{1}=[0, e], L_{2}=\left[0, e^{*}\right]$. Since $L \cong L_{1} \times L_{2}$, it follows that $G \cong G_{e} \times G_{e^{*}}$, where $G_{e}$ and $G_{e^{*}}$ are the convex $\ell$-subgroup of $G$ generated by $e$ and $e^{*}$, respectively. In fact, $G$ is the direct sum of $G_{e}$ and $G_{e^{*}}$, because $G_{e} \cap G_{e^{*}}=\{0\}$ and $G_{e}+G_{e^{*}}=G$. The MV-algebra $\Gamma\left(G_{e}, e\right)$ is just the interval algebra $L_{e}=[0, e]$. Also, from Proposition 4.17, $d$ can be extended to a morphism, denote $\overline{\mathrm{d}}$, from $(G, u)$ onto $\left(G_{e}, e\right)$. It is not hard to show that $\overline{\mathrm{d}}$ agrees with the projection of $G$ as $G_{e}$ as a direct summand.

Conversely, if $d$ is a regular difference derivation on MV-algebra $L$ such that $e=d(1) \in B(L)$, then follows from Corollary 4.19 that $L \cong L_{1} \times L_{2}$, where $L_{1}=\left([e, 1], \oplus, \star_{3^{\prime}}, e\right), L_{2}=\left(\left[e^{*}, 1\right], \oplus, \star_{4^{\prime}}, e^{*}\right)$.

\section{Conclusions}

The notion of derivations is helpful for studying structures in algebraic systems. In this paper, we give some representations of MV-algebra in terms of derivations. In particular, we obtain that every Boolean algebra is isomorphic to the algebra of all implicative derivations and prove that regular difference derivations coincide with direct product decompositions of MV-algebras. Then, we obtain that a regular derivation pair $(d, g)$ forms a Galois connection on $L$ if and only if $g(L)=d(L)$. Since the above topics are of current interest we suggest further directions of research:

(1) we will further discuss the relationship between Galois connections and regular derivation pairs on MV-algebras and solve the open problem as we mentioned in the Section 4.

(2) since regular derivation pairs and Galois connections are closely related, the latter plays an important role in studying Formal Concept Analysis [8]. In the future, we will find the application of regular derivation pairs in Formal Concept Analysis .

\section{Acknowledgments}

The authors are extremely grateful to the editors and the referees for their valuable comments and helpful suggestions which help to improve the presentation of this paper. This study was funded by a grant of National Natural Science Foundation of China (61472471,11801440), the Innovation Talent Promotion Plan of Shaanxi Province for Young Sci-Tech New Star (2017KJXX-60), 
Natural Science Foundation of Education Committee of Shannxi Province (19JK0653).

\section{References}

[1] N. O. Alshehri, Derivations of MV-algebras, International Journal of Mathematics and Mathematical Sciences, 2010(2010)1-8.

[2] R. A. Borzooei, O. Zahiri, Some results on derivations of BCI-algebras, Scientiae Mathematicae Japonicae, 26(2013)529-545.

[3] C. C. Chang, Algebraic analysis of many-valued logic, Transactions of the American Mathematical Society, 88 (1958)467-490.

[4] C. C. Chang, A new proof of the completeness of the Eukasiewicz axioms, Transactions of the American Mathematical Society, 93 (1959)74-80.

[5] R. Cignoli, D. Mundici, An elementary proof of Chang's completeness theorem for the infinite-valued calculus of Łukasiewicz, Studia Logica, $\mathbf{5 8}$ (1997)79-97.

[6] R. Cignoli, I.M.L. D'Ottaviano, D. Mundici, Algebraic Foundations of Many-valued Reasoning, Kluwer Academic Publishers, Dordrecht, 2000.

[7] F. Esteva, L. Godo, Monoidal t-norm based logic: towards a logic for left-continuous t-norms, Fuzzy Sets and Systems, 124 (2001) 271-288.

[8] B. Ganter, R. Wille, Formal Concept Analysis, Mathematical Foundations, Springer-Verlag, Berlin, 1999.

[9] T. S. Blyth, Lattices and ordered algebraic structures, Springer-Verlag London Limited, 2005.

[10] P. Hájek, Metamathematics of Fuzzy Logic, Kluwer Academic Publishers, Dordrecht, 1998.

[11] P. F. He, X. L. Xin, J. M. Zhan, On derivations and their fixed point sets in residuated lattices, Fuzzy Sets and Systems, 303 (2016) 97-113.

[12] Y. B. Jun, X. L. Xin, On derivations of BCI-algebras, Information Sciences, 159 (2004) 167-176.

[13] S. D. Lee, K. H. Kim, On derivations of lattice implication algebras, Ars Combinatoria, 108(2013) 279-288. 
[14] J. Liang, X. L. Xin, J. T. Wang, On derivations of EQ-algebras, Journal of Intelligent and Fuzzy Systems, 35(2018) 5573-5583.

[15] D. Mundici, Mapping abelian $\ell$-groups with strong unit one to one into MV-algebras, Journal of Algebra,98 (1986) 76-81.

[16] E. Posner, Derivations in prime rings, Proceedings of the American Mathematical Society, 8 (1957) 1093-1100.

[17] E. Turunen, Mathematics Behind Fuzzy Logic, Physica-Verlag, Heidelberg, 1999.

[18] J. T. Wang, A. Borumand Saeid, M. Wang, On derivations of commutative multiplicative semilattices, Journal of Intelligent and Fuzzy Systems, 35(2018) 957-966.

[19] X. L. Xin, The fixed set of aderivation in lattices, Fixed Point Theory and Applications, 218(2012) 1-12.

[20] X. L. Xin, T. Y. Li, J. H. Lu, On derivations of lattices, Information Sciences, 178(2008) 307-316.

[21] H. Y. Yong, On $f$-derivations of lattice implication algebras, Ars Combinatoria, 110(2013) 205-215.

[22] H. Zhu, Y. Liu, Y. Xu, On derivations of linguistic truth-valued lattice implication algebras, International Journal of Machine Learning and Cybernetics, 9(2018) 611-620.

[23] J. M. Zhan, Y. L. Liu, On f-derivations of BCI-algebras, International Journal of Mathematics and Mathematical Sciences, 11(2005) 1675-1684.

Jun Tao Wang,

School of Science,

Xi'an Shiyou University,

Xi'an, 710065, Shaanxi, China.

Email: wjt@xsy.edu.cn

Yan Hong She,

School of Science,

Xi'an Shiyou University,

Xi'an, 710065, Shaanxi, China.

Email: yanhongshe@yeah.net

Ting Qian,

School of Science,

Xi'an Shiyou University,

Xi'an, 710065, Shaanxi, China.

Email: qiant2000@126.com 